\title{
Infrared Prism Spectrometry from 24 to 40 Microns*
}

\author{
By Earle K. Plyler
}

\begin{abstract}
A prism of KRS-5 (thallium-bromide-iodide) has been used to measure the transmission of various substances in the infrared region from 24 to 40 microns. The prism was installed in a Perkin-Elmer spectrometer, and a Golay detector was employed for measuring the energy. Transmission data were obtained for polystyrene, polyethylene, and different samples of KRS-5. Also measurements have been made on the cut-off of $\mathrm{KBr}, \mathrm{AgCl}$, and other crystals. The rotational spectrum of the water-vapor molecule has been measured to 39.2 microns, and the known frequencies of these absorption bands have been used for the calibration of the prism.
\end{abstract}

\section{Introduction}

Some of the optical properties of the thallium halides have been known for a considerable time. The residual rays of some of these substances were measured by Rubens and Wartenberg ${ }^{1}$ in 1914. The maxima of the reflection bands were $91.9 \mu$ for $\mathrm{TlCl}, 117 \mu$ for $\mathrm{TlBr}$, and $152 \mu$ for TII. These reflection maxima fall in the long wavelength region of the infrared. This fact indicated that the absorption of the material would not be intense for moderate thicknesses to about 40 or $50 \mu$. Thallium iodide does not form cubic crystals and usually exists as a powder. In order to use it for infrared studies, a mixed crystal with TlBr from the fused mixture is grown. During the war such mixed crystals were grown in Jena. The material was called KRS-5 and consisted of about 42 percent of TlBr and 58 percent of TII. A mixed crystal of $\mathrm{TlCl}$ and $\mathrm{TlBr}$ was called KRS-6 and other materials that were used in the infrared region were also included in the KRS series. The KRS-5 is highly resistant to water and was found useful on field instruments. Preliminary measurements in Germany showed that the TIBr-I crystal has a high index of refraction in the visible and the infrared regions and that in thicknesses of $2 \mathrm{~mm}$ it is transparent beyond $40 \mu$.

In 1945 plans were made at Fort Belvoir and at the National Bureau of Standards for the growing of synthetic crystals of the thallium

\footnotetext{
${ }^{1}$ H. Rubens and H. V. Wartenberg, Berl. Ber (1914).

*Presented in part at the meeting of the Optical Society of America, Mar. 6, 1948; J. Opt. Soc. Am. 38, 664 (1948).
}

halides. At first, considerable difficulty was experienced in producing single crystals of sufficient size for prisms. Also many of the early crystals became opaque beyond $30 \mu$. This difficulty was overcome when great care was takn in purifying the thallium bromide and thallium iodide.

\section{Experimental Procedure}

A single crystal of KRS-5 was grown by Francis Phelps of this Bureau, and from it a prism with a refracting angle of $26^{\circ}$ was cut and polished in the Optical Shop. The prism was annealed before the final polishing. Some experiments on the polishing were carried out by Edgar Robinson, and he was able to get good surfaces that are flat to a few wavelengths of monochromatic yellow light over a considerable part of the prism face. The refracting angle of $26^{\circ}$ was selected, in part, so that the prism could be used in a Perkin-Elmer spectrometer with a minimum of changes in the instrument. The KRS-5 has a high dispersion in the visible region, and the yellow mercury lines are well separated. It is possible to obtain an image of about 0.25 $\mathrm{mm}$ of a single visible spectral line by using an extremely narrow entrance slit.

The difficulty of obtaining very narrow images appears to be due to lack of homogeneity of the prism. If the composition of the crystal varies in such a manner that the proportions of $\mathrm{TlBr}$ and TII differ for different regions within the crystal, corresponding variations in the index of refraction will result for the same regions. A 
prism, obtained from another laboratory that was recently examined, produced four images of a single spectral line. The lack of sharpness of the image is not of great importance in the region from 25 to $40 \mu \quad$ Because of the small amounts of energy radiated by the globar in this region, it is necessary to use slits up to $1 \mathrm{~mm}$.

A Golay detector with a $\mathrm{KRS}-5$ window was used as the deterting unit. The energy from the exit slit was focused on the blackened receiver by means of a lens made from KRS-5.

The energy is further reduced by the reflection losses of the prism. For example, at $28 \mu$ the index of refraction is about 2.3 , and a $26^{\circ}$ prism loses about 50 perce it of the energy by reflection when it is traversed twice. Under the same conditions, a $36^{\circ}$ prism loses about 72 percent of the incident energy by reflection. Small scratches on the surface of the prims do not modify its properties for long wavelength energy to any great extent but may produce scattering of radiation in the short wavelengths.

\section{Results}

The infrared spectrometer, containing the KRS-5 prism as the dispersing unit, was calibrated for wavelength determinations by the use of the known absorption bands of many substances in the region from 2 to $40 \mu$. A graph was made by plotting the drum readings of the spectrometer as a function of the wavelength. On account of the low dispersion of the KRS-5 prism in the region from 2 to $15 \mu$, it is not advantageous to use a prism of this material for this region. Good resolution is obtained in the region from 24 to $40 \mu$, and a more detailed calibration curve has been obtained for this range of wavelengths. By the positions of the absorption bands of chloroform, bromoform, carbon disulfide, and carbon tetrachloride, the rotation lines of the water vapor absorption spectrum were identified. The wave numbers of these lines in the 24 - to $40-\mu$ region are well known from the work of Randall, Dennison, Ginsburg, and Weber. ${ }^{2}$ Their work was carried out with a grating spectrometer, and the resolution is much higher than that obtained with the KRS-5 prism. However, many of the absorption lines are single and show up distinctly on the recorder traces. Where some of the lines

\footnotetext{
${ }^{2}$ H. M. Randall, D. M. Dennison, N. Ginsburg, and L. R. Weber, Phys. Rev. 52, 160 (i937).
}

of the water vapor molecule are not separated by the KRS-5 prism, an average value is used for the region of maximum absorption. The calibration curve is shown in figure 1 . The graph was drawn through the experimentally determined wavelengths, and a smooth curve was obtained. The dispersion of the prism in this region is very good, six and one half turns of the spectrometer drum being necessary to change from 22 to $39 \mu$.

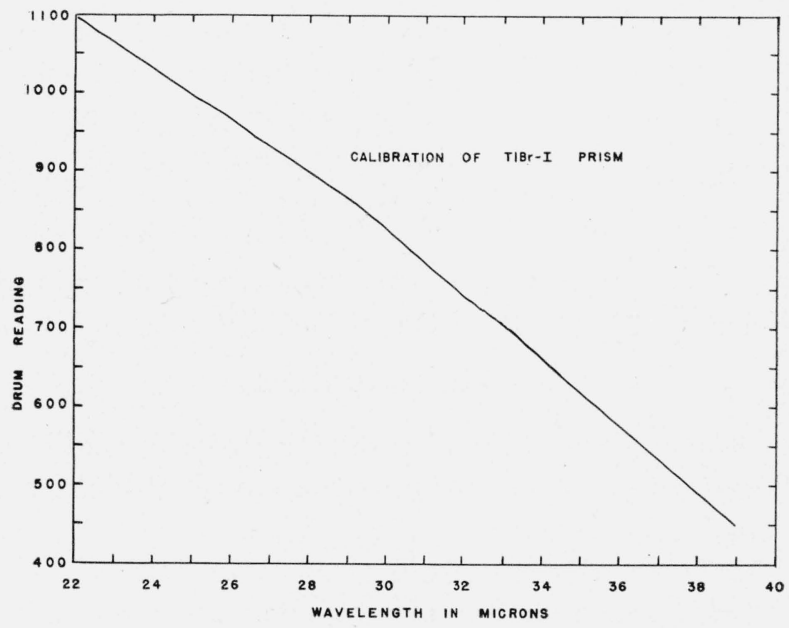

FIgURE 1. Calibration curve for the KRS-5 prism used in a Perkin-Elmer infrared spectrometer.

In the measurement of the water vapor spectrum, stray radiation became quite appreciable at $34 \mu$, and increased to about 65 percent of the total energy at $40 \mu$. In figure 2 is shown the water vapor spectrum as measured with an aluminum reflector in the source box. The two lines that are resolved in the region of $30.6 \mu$ are separated by $4 \mathrm{~cm}^{-1}$. The spectral slit width of the instrument using slits of $0.5 \mathrm{~mm}$ is much less than $4 \mathrm{~cm}^{-1}$, and the practical limit is about $2 \mathrm{~cm}^{-1}$.

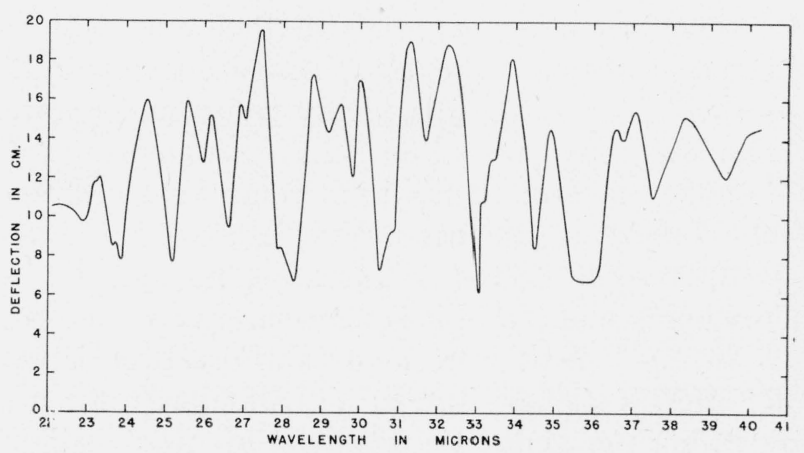

Figure 2. Absorption spectrum of water vapor measured with an aluminum mirror in the source box of the spectrometer. 
In figures 3 and 4 are shown the water vapor spectrum for the same region using reflectors of $\mathrm{CaF}_{2}$ and $\mathrm{NaF}$. The stray radiation is reduced to about 5 percent at $37 \mu$, but there is also a reduction of the total energy. It is possible to extend the measurements with a KRS-5 prism to beyond $40 \mu$, but the energy is greatly reduced. Small amounts of energy are transmitted by the prism to $41 \mu$, but only an approximate measurement of the transmission of substances can be made at that wavelength.

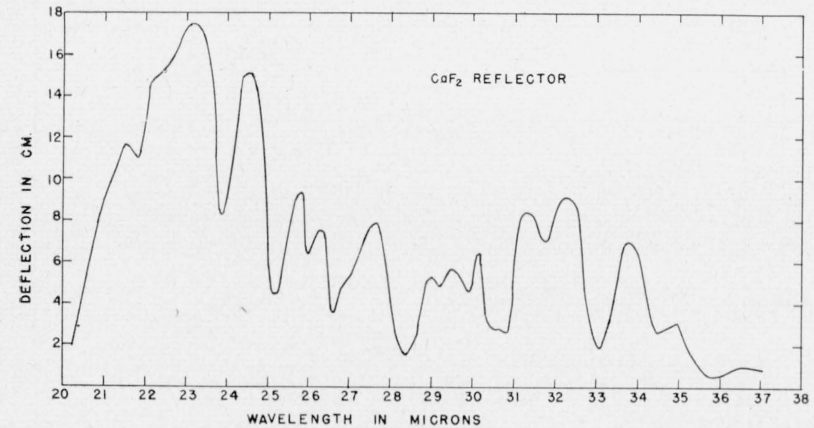

FiguRE 3. Absorption spectrum of water vapor measured. with a $\mathrm{CaF}_{2}$ reflector.

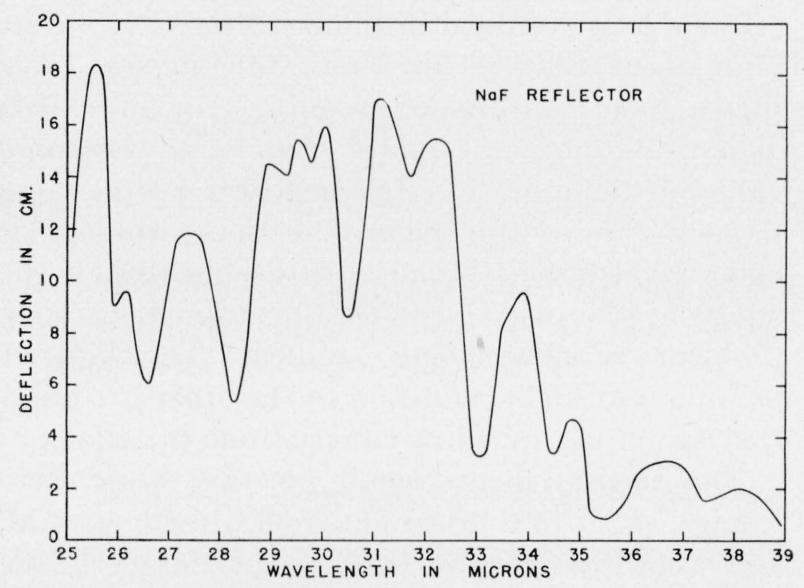

FiguRE 4. Absorption spectrum of water vapor measured with a NaF reflector.

The infrared spectrometer with the KRS-5 prism and filters has been used to measure the infrared transmission of different solids. Other materials were sought that would be transparent in the 24 to $40 \mu$ region. In figure 5 is shown the percentage transmission of polystyrene, polyethylene, KRS-5, KBr, and $\mathrm{AgCl}$. Polystyrene shows the highest transmission in this region of any substance measured. The high transparency is partly caused by the fact that the layer is only $25 \mu$ thick. When a $1-\mathrm{mm}$ window was used, only 25 percent of the incident radiation is transmitted at $33 \mu$. However, for coatings and windows, thin films of polystyrene are useful. A layer of polyethylene $240 \mu$ in thickness also shows high transmission in this region. Thinner layers show somewhat higher transmission. The irregular form of the transmission curve is probably caused by absorption in the polyethylene. Films of $\left(\mathrm{C}_{2} \mathrm{~F}_{4}\right)_{\mathrm{x}}-$ $\left(\mathrm{C}_{2} \mathrm{H}_{2} \mathrm{~F}_{2}\right)_{\mathrm{x}}$, and $\left(\mathrm{C}_{2} \mathrm{H}_{2} \mathrm{Cl}_{2}\right)_{\mathrm{x}}$ were also measured, but they have low transmission in this region. The $\mathrm{KBr}$ sample is $6.4 \mathrm{~mm}$ thick and transmits about 10 percent at $31.5 \mu$. Thus, in thicknesses of $1 \mathrm{~mm}$ or less, $\mathrm{KBr}$ will be useful as a window to $30 \mu$. A sheet of $\mathrm{AgCl}, 2.1 \mathrm{~mm}$ thick, transmits 10 percent at $27.5 \mu$. However, the sample is slightly discolored and may not have as high transmission as a clear specimen.

Many samples of KRS-5 have been examined, and in figure 5 is shown the transmission of a clear specimen. The transmission at $24.5 \mu$ is approximately 70 percent, about the same as at $20 \mu$. Most of the loss of energy is caused by reflection. As the wavelength is increased, the transmission decreases and is about 45 percent at $37 \mu$.

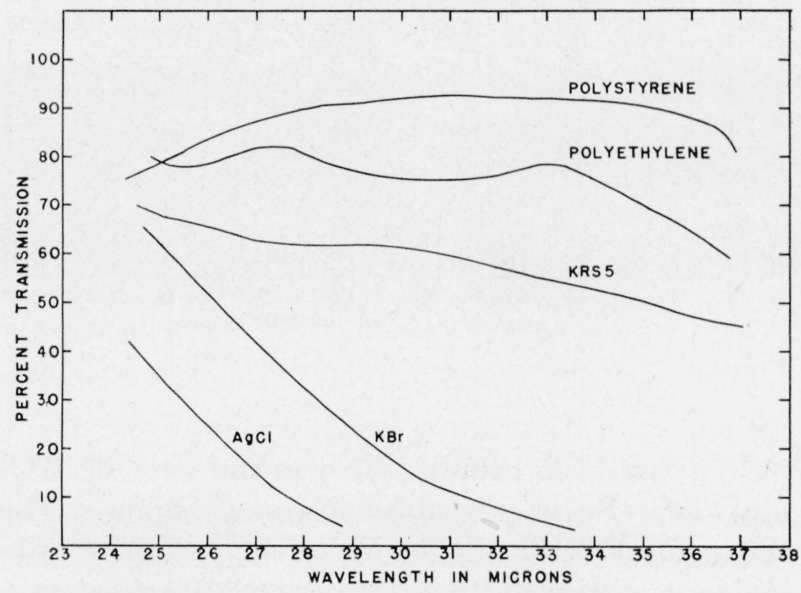

Figure 5. Transmission of polystyrene 25 microns thick, polyethylene 240 microns, KRS 5 \& $\mathrm{mm}, \mathrm{KBr} 6.4 \mathrm{~mm}$, and $\mathrm{AgCl} 2.1 \mathrm{~mm}$.

In figure 6 is shown the transmission of other samples of KRS-5. The lower curve is for a sample that probably contained impurities. One sample, for which the measurements are not plotted on the graph, became opaque at $26 \mu$.

In figure 7 is shown the transmission of four chlorides. The $\mathrm{PbCl}_{2}$ and $\mathrm{TlCl}$ samples were obtained from the Naval Research Laboratory and 


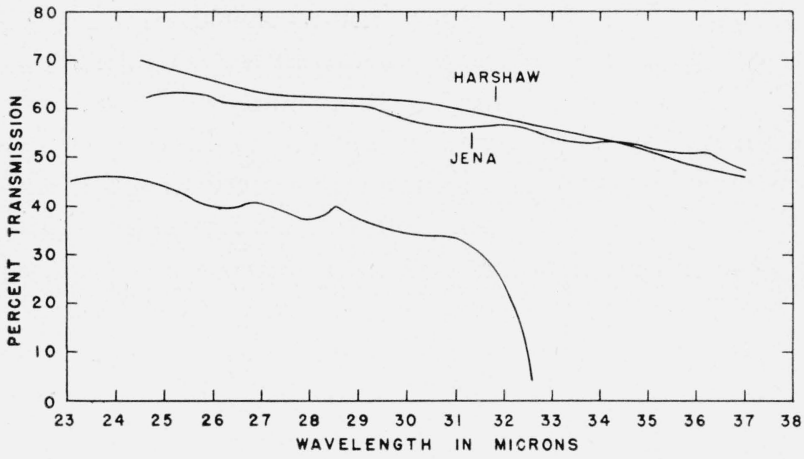

Figure 6. Transmission of three different samples of KRS-5, thickness $8 \mathrm{~mm}$.

the $\mathrm{AgCl}$ and $\mathrm{KCl}$ samples from Harshaw Chemical Co. All of these materials are well suited for window materials, but the $\mathrm{AgCl}$ is the only one of these substances that is not attacked by water.

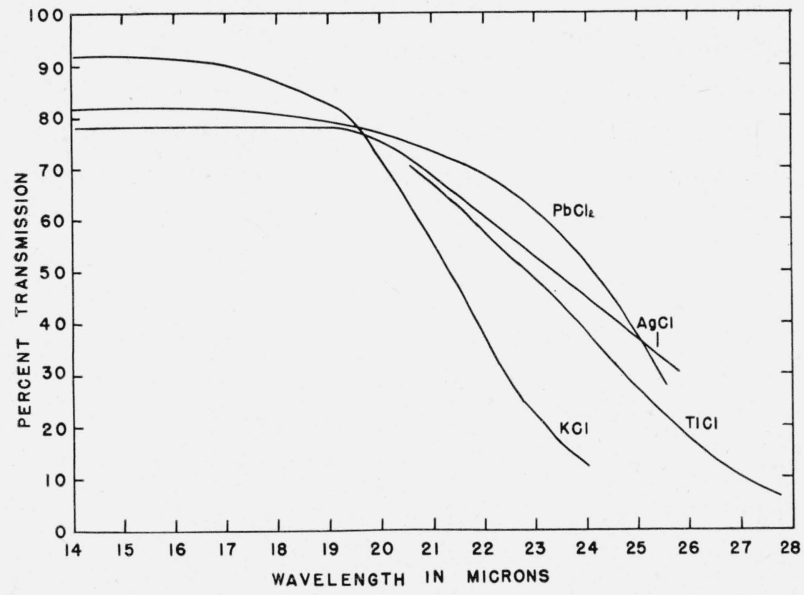

Figure 7. Transmission of $\mathrm{PbCl}_{2} \mathrm{KCl}, \mathrm{TlCl}$, and $\mathrm{AgCl}$, $6 \mathrm{~mm}$ in thickness.

In figure 8 is shown the transmission of $\mathrm{KCl}$ and $\mathrm{TlCl}-\mathrm{Br}$ in the near infrared region. The $\mathrm{KCl}$ sample transmitted about 92 percent of the incident energy at $16 \mu$. The $\mathrm{TlCl}-\mathrm{Br}$ reached a value of about 80 percent at $12 \mu$.

In figure 9 , the transmission of four thallium halides is given. The two samples of TlCl-Br were obtained from different sources. The samples were not annealed and that accounts, in part, for their irregular transmission curves. Some of the small dips in the graph may represent impurities in the sample. Also impurities may cause the different transmitting properties of the two samples of TlCl-Br.

The technique of growing the thallium halide

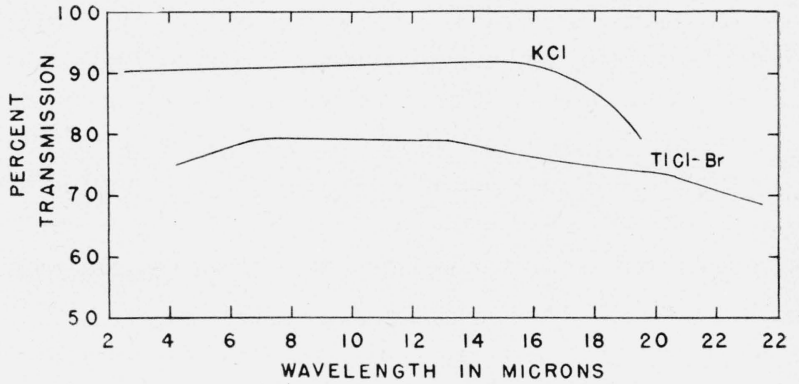

Figure 8. Transmission of $\mathrm{KCl}$ and $\mathrm{TlCl}-\mathrm{Br}, \mathrm{KCl}, 12$ $\mathrm{mm}$ in thickness, TlCl-Br, $6 \mathrm{~mm}$ in thickness.

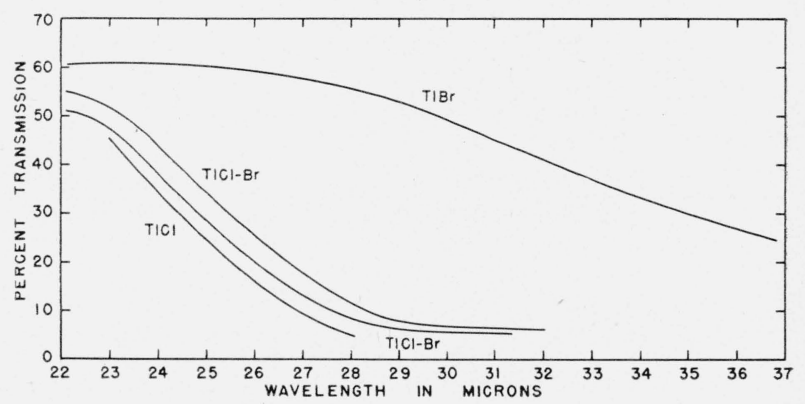

Figure 9. Transmission of TIBr, of 2 different samples of $\mathrm{TlCl}-\mathrm{Br}$ and of $\mathrm{TlCl}$.

All the samples were approximately $6 \mathrm{~mm}$ in thickness.

crystals has improved in the last year, and recent samples of KRS-5, in 6-mm thicknesses, have shown good transmission to $40 \mu$. However, it is difficult to obtain samples that have the same value for the index of refraction over a large cross section. Some transparent sections would not make good prisms because of the blurring of the image at the exit slit of the spectrometer. This problem is now being studied. The KRS-5 prism is a valuable addition to the other materials that are in use for infrared spectrometry.

The measurements made in this work were carried out with a prism cut from a single crystal, and it has been possible to obtain partial resolution of water-vapor lines separated by $2 \mathrm{~cm}^{-1}$. However, in actual operation the spectral slit widths are about $4 \mathrm{~cm}^{-1}$ at $33 \mu$. In the region from 23 to $25 \mu$, better resolution is obtained with the KRS-5 than with the $\mathrm{KBr}$ prism. The lower resolution of the $\mathrm{KBr}$ prism in this region probably results from absorption by the $\mathrm{KBr}$ prism at $23 \mu$ and longer wavelengths.

Further studies on the transmission of liquids and vapors in the 24 - to $40-\mu$ region are being carried out and will be reported in a future paper.

Washington, May 11, 1948. 